\title{
The Analysis of Settings of the Material Prime Cost in BOQ Tender
}

\author{
Jianfeng $\mathrm{Li}^{1,2, a}$, Bingliang Wang ${ }^{1, \mathrm{~b}}$ and Xiaoju Cao ${ }^{1, \mathrm{c}}$ \\ ${ }^{1}$ School of Civil and Engineering, Chang'an University, Xi'an 710061, China \\ ${ }^{2}$ Engineering Technology Department, Xijing University, Xi'an 710123, China \\ alilaoshi1956@163.com, b912270351@qq.com, ' $1141058674 @ q q . c o m$
}

Keywords: The material prime cost setting; Significance; Defects; the decision method.

\begin{abstract}
It will help improve its results to set the material prime cost, but producing defects that it is not conducive to full competition, the cost management, risk sharing and cost control. This paper points out the problem of whether to set the prime cost for a material that tenderers face. And put forward a decision method to solve the problem through explaining its principle, analyzing its construction and exemplifying its application.
\end{abstract}

\section{Introduction}

Since prime cost sum was formally put forward in code of valuation in 2008, research on the analysis of the pros and cons and management recommendations to the material prime cost has been conducted by many scholars, meanwhile some scholars propose that prime cost sum should not be set too much ${ }^{[1]}$. However, rational decision methods are lacking when deciding whether to set prime cost for a material whose price is difficult to determine. The author tries to put forward a relatively quantitative decision method to solve the decision problem on the material (including engineering equipment) prime cost settings for tenderers to some extent.

\section{Significances and Defects of Setting the Material Prime Cost}

Be conducive to improve the tendering result. The bidders will be unable to bid on the materials' price, avoiding the malicious competition caused by the standard uncertainty, which is conducive to select the bidders of real strength for the tenderers.

Be not conducive to the full competition and not risk sharing. The employer will assume all the price increasing risk of the materials set prime cost because the materials' cost will be adjusted according to their price confirmed in the construction implementation phase according to the 13 edition of "Code of bills of quantities and valuation for construction works" ${ }^{22]}$. Also, be not conducive to cost control, especially in the state-owned investment projects. At present, some areas have introduced the relevant documents, making the upper limit provisions to prime cost sum. For instance, Beijing city stipulates the proportion of both the prime cost sum and the tentative engineering sections must be not more than $30 \%$ of the contract sum ${ }^{[3]}$. Nantong city makes regulations to the upper limit proportion of prime cost sum in tender sum limit for the state-owned projects ${ }^{[4]}$.

\section{The Necessity of the Material Prime Cost Setting Decision Method}

Rational decision method better reconstructs the value of the material prime cost. Tenderers are likely to set prime cost for materials whose price is difficult to determine but having small effect on the tendering result. Similarly, materials having large effect on the tendering result are also likely to not be set prime cost for (as shown in the dotted arrow part of Fig. 1). Rational decision method will make the material prime cost better play its significance and avoid its defects, when used by tenderers (as shown in the solid arrow part of Fig. 1). 


\section{The Decision Method of Setting the Material Prime Cost}

The principle of the decision method of setting the material prime cost (as shown in Fig. 2). If the maximum quotation difference for a material whose price is difficult to determine among all bidders is less than the minimum tendering sum difference among all bidders, we can think that it will not affect the tendering sum order and not affect the tendering result. Therefore, tenderers can decide whether to set the prime cost for a material whose price is difficult to determine according to the expected quotations of all bidders for the materials.

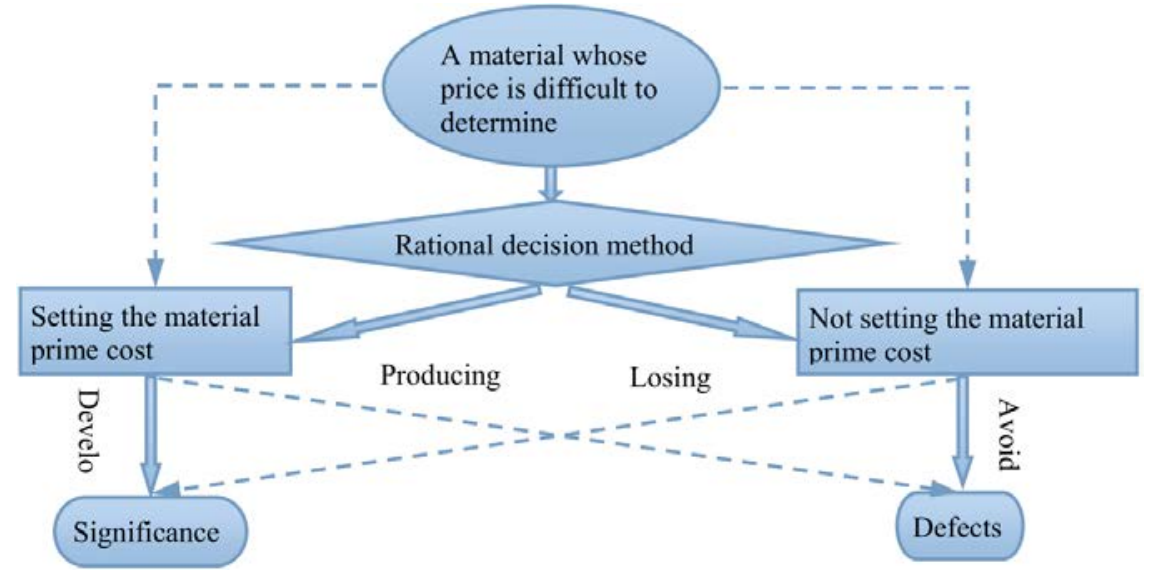

Fig. 1 Rational decision method better reconstructing the value of the prime cost

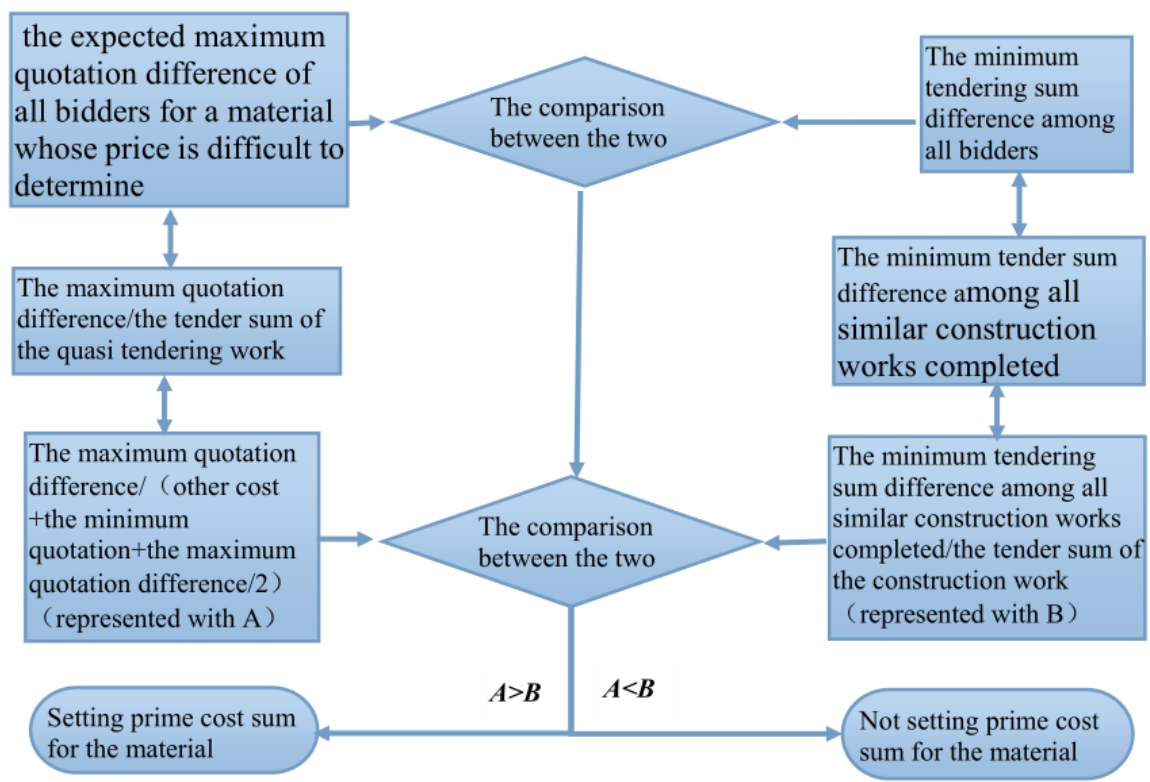

Fig. 2 The principle of the decision method of settings of the material prime cost

(1) The determination of the minimum tendering sum difference among all bidders. The minimum tendering sum difference among all bidders is unknown when tendering and the data can be concluded according to similar construction works completed (it should be considered that similar construction works completed are the same as the quasi tendering work on the using function of buildings and structure), conservatively, taking the minimum tendering sum difference among all similar construction works completed. The minimum tendering sum difference among all similar construction works completed has a chance, so thinking that it obeys the normal distribution.

(2) The relative processing of the minimum tendering sum difference among all bidders. In order to improve the reliability, the absolute index, the minimum tendering sum difference, can be replaced by the relative index, the percentage of the minimum tendering sum difference in the tender sum limit. 
Similarly, the absolute index, the maximum quotation difference for a material whose price is difficult to determine in the quasi tendering work, can be replaced by its percentage in the tender sum of the quasi tendering work.

(3) The determination of the tender sum of the quasi tendering work. Under the circumstance that the price of a material is difficult to determine, the tender sum of the quasi tendering work can be divided into the material cost and other cost. The material cost can be replaced by the tenderers-expected average quotation of all bidders for the material, the average quotation approximately equal to the minimum quotation plus half of the maximum quotation difference.

Constructing the inequality decision method of setting the material prime cost.

$X$ — the expected minimum quotation of all bidders for a material whose price is difficult to determine;

$Y$ — the expected maximum quotation difference of all bidders for a material whose price is difficult to determine;

$Z$ — other cost of the quasi tendering work other than the cost of the material whose price is difficult to determine;

$M$ — the percentage of the minimum tendering sum difference among all similar construction works completed in the tender sum of the construction work;

$M_{\text {min }}$ - the minimum percentage of the minimum tendering sum difference among all similar construction works completed in the tender sum of the construction work;

$p$ — the guarantee rate of the minimum percentage of the minimum tendering sum difference among all similar construction works completed in the tender sum of the construction work.

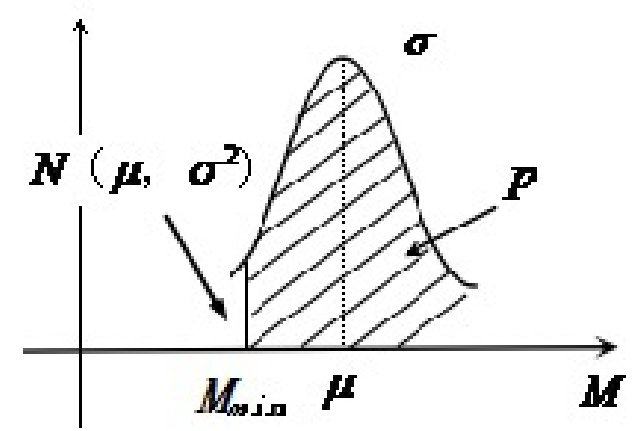

Fig. 3 The normal distribution

Because $M \sim N\left(\mu, \sigma^{2}\right)$, so $\frac{M-\mu}{\sigma} \sim \Phi(x)$.Make $\Phi\left(x_{0}\right)=1-p$, so $P\left(\frac{M-\mu}{\sigma} \leq x_{0}\right)=1-p$, i.e. $P\left(M \leq \sigma x_{0}+\mu\right)=1-p$, which is to say $M_{\min }=\sigma x_{0}+\mu$ whose guarantee rate is $p$, approximately, $\mu=\bar{M} \sigma^{2}=S^{2}=\frac{1}{n-1}\left(\sum_{i=1}^{n} M_{i}^{2}-n \bar{M}^{2}\right)$ as shown in Fig. 3.

Under the circumstance that the material is not set the prime cost for, if $\quad$ i.e. $\left(1-0.5 M_{\min }\right) Y-M_{\min } X<M_{\min } Z$, so it has little effect on the tendering result not to $\frac{Y}{Z+X+\frac{Y}{2}}<M_{\min }$

set the prime cost for the material whose price is difficult to determine. Otherwise, significant effect, the prime cost should be set.

\section{An Example of the Application of the Inequality Decision Method of Setting the Material Prime Cost}

Other cost of a quasi-tendering work other than the cost of the material whose price is difficult to determine, $\mathrm{Z}$, is $¥ 8,000,000$, the expected minimum quotation of all bidders for a material whose 
price is difficult to determine Tithe expected maximum quotation difference $\mathrm{Y}, p=0.75$. Through statistically calculating according to relevant cost documents of similar construction works completed, know $M=(1 \%, 2 \%, 3 \%, 4 \%, 5 \%, 6 \%, 7 \%, 8 \%, 9 \%, 10 \%)$.

An After calculations $\bar{M}=5.5 \% S^{2}=(3.03 \%)^{2} . M_{\min }=\sigma x_{0}+\mu$ whose guarantee rate is 0.75 , approximately, $\mu=\bar{M}, \sigma=S$, so $M_{\text {min }}=3.46 \%$, i.e. the probability is $75 \%$ to assure $3.46 \%$ is the minimum percentage of the minimum tendering sum difference among all similar construction works completed in the tender sum of the construction work, as shown in Fig. 4.

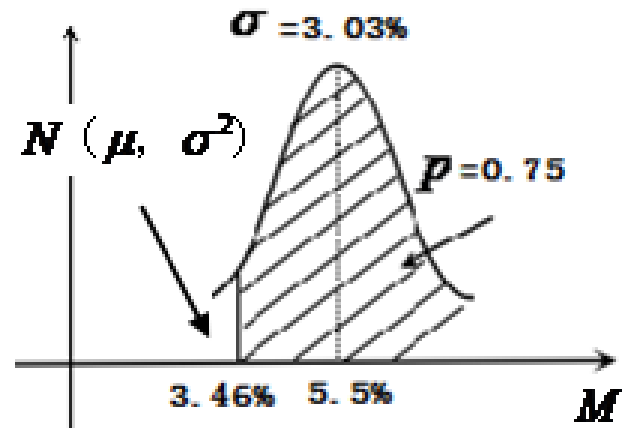

Fig. 4 The normal distribution

Under the circumstance that the material is not set the prime cost for, make $\frac{Y}{800+X+\frac{Y}{2}}<3.46 \%$, i.e. $0.9827 Y-0.0346 X<27.68$. If the tenderer-expected $\mathrm{X}$ is $¥ 1000,000, Y ¥ 300,000$, then put them into the left part of the inequality, $0.9827 \times 30-0.0346 X=26.02<27.68$. Therefore, there is no need to set the prime cost for the material whose price is difficult to determine.

\section{Summary}

In summary, advantages and disadvantages appeared are different in tendering activities whether to set the prime cost for a material whose price is difficult to determine. And the use of rational decision method to judge will help tend to advantages and avoid disadvantages. The inequality decision method can be an important reference for tenderers setting prime cost for materials which is constructed according to the expected minimum quotation of all bidders for a material whose price is difficult to determine, the maximum quotation difference and the percentage of tendering sum among all similar construction works completed in the tender sum.

\section{References}

[1] Jiefeng Lv. Discussions of prime cost sum under the model of bills of quantities and valuation. Construction Economy, 2013, (1): p.19-21.

[2] Ministry of Housing and Urban-Rural Construction of the People's Republic of China. GB50500-2013, Code of bills of quantities and valuation for construction works. Beijing: China Planning Press, 2013: p.18.

[3] Beijing Municipal Commission of Housing and Urban-Rural Development. Circular on further regulating the bidding activities for housing and public infrastructure construction in Beijing. 2011-03-31. http://www.bjjs.gov.cn/publish/portal0/tab662/info62263.htm.

[4] Nantong City Urban-Rural Construction Bureau, Nantong City Supervision Bureau, Nantong City Finance Bureau, etc. Regulations on management of prime cost sum for construction works and materials, plant and equipment supplied by Employer (Trial). 2011-1-30. http: //haggy. He-men.gov.cn/herb/Info Detail/? Info ID= 6c9b85fd-1ca2 -4da7-983c- ea12e3d05c31 \& Cate gory Num $=003004$. 